\title{
Peering into the Abyss with Evolutionary Telescopes: How protein structure-telescopes can look further back in time (and why sequence- telescopes cannot)
}

\author{
Ajith Harish \\ Independent Researcher, Uppsala, 756 57, Sweden
}

\begin{abstract}
This year marks the Golden Jubilee celebrations of the Protein Data Bank (PDB), which catalogs three-dimensional (3D) shapes of organic macromolecules and showcases a structural view of biology. In celebrating this occasion, much has been written about the transformative impact of PDB on a broad range of fields of scientific inquiry and how structural biology transformed the study of the fundamental processes of life. Yet, the transforming influence of PDB on one field of inquiry of broad interest - the reconstruction of the distant evolutionary past - has gone almost unnoticed. Here, I review recent advances to highlight how insights and tools of structural biology are bearing on the data required for the empirical resolution of vigorously debated and apparently contradicting theories in evolutionary biology.
\end{abstract}

\section{Introduction}

The linear amino acid chains of most proteins are physically folded into a specific 3D structure to become stable and functional. Misfolded proteins are usually non-functional and degraded but can often be lethal to the cell. The Protein Data Bank (PDB), established in 1971 with a handful of protein structures determined by X-ray crystallography, is one of the first opensource data repositories (Bank, 1971). The PDB now hosts more than 180,000 structures of proteins, nucleic acids and assemblies of supramolecular complexes. The PDB has transformed many life sciences disciplines by enriching our understanding of the physical and chemical basis of the fundamental biological processes. Article collections recount how PDB changed biology and celebrate the $50^{\text {th }}$ anniversary of PDB (Gierasch \& Berman, 2021; Zardecki et al., 2021). On the heels of the publication of the special issues celebrating the $50^{\text {th }}$ anniversary of $\mathrm{PDB}$, the latest computational tools for de novo structure prediction joined the celebratory bandwagon.

Considered to be a Once in a generation advance, the latest computational tools such as Alphafold (Tunyasuvunakool et al., 2021) and RoseTTaFold (Baek et al., 2021) are a major leap in de novo protein structure prediction. De novo structure prediction from protein sequences that have no representative structures is a hard problem. The new algorithms extract information in protein sequences that are "trained" for eons by evolution for spontaneous folding into specific and complex 3D shapes. The significance of these new algorithms compared to their predecessors is (a) the speed of determining the best folded conformation of a given linear amino acid sequence among the numerous possible 3D conformations, and (2) the unmatched accuracy of the predicted structure, which is as good as the structures determined by X-ray crystallography and other experimental methods (Baek et al., 2021; Tunyasuvunakool et al., 2021). 
Tools such as Alphafold and RoseTTaFold are a shot in the arm in the efforts to map the "protein universe". The protein universe is the assortment of all proteins from all organisms that have evolved on Earth over $\approx 3.8$ billion years (Levitt, 2009). Whether or not such computational de novo structure predictions can replace the many experimental methods is an intriguing prospect for the future. At any rate, undeniably, the success of the algorithmic predictions and the boost in their predictive power relies on the thousands of experimentally vetted structures available in the PDB. The training data from which the rules and physicochemical constraints of protein folding are learnt underscores the invaluable insights gained from these high-resolution structural data.

The availability of the large number of protein structures has significantly improved the efforts in resolving another hard problem - reconstructing evolution itself. Specifically, looking back into the earliest stages of cellular evolution through "evolutionary telescopes". The tremendous advantages of a protein structure-based evolutionary telescope (i.e., a phylogeny) over its predecessor - the more commonly used sequence-based telescope, is going almost unnoticed. In celebrating the transformative influence of the PDB and structure-based insights on resolving a myriad of biological problems, this essay puts the spotlight on the impact of structural biology on dissecting longstanding problems in evolutionary biology.

\section{Evolution of the protein universe recapitulates the evolution of cellular universe}

The vast majority of organismal species is microbial (Locey \& Lennon, 2016), that is they are single-celled organisms. Cells can be seen as membranous ensembles studded with proteins inside and out (Figure 1). Proteins are components of the molecular machinery involved in all cellular functions (Figure 1A), from the birth through death of cells. Proteins are not only the workhorses of cells that drive the molecular machinery, but they also make up the infrastructure that maintains the morphology and internal organization of cells (Figure 1B). Enzymatic proteins that catalyze the biochemical reactions are an example of the former and cytoskeletal proteins of the latter. Based on the extent of membranous organization observed in ultrastructures of cells, two basic types of cellular organisms are known (Figure 1C),

- Eukaryotes (Greek; eu, "well" and karyon, "kernel"): Organisms with a well-defined membrane bound nucleus and other membrane bound intracellular compartments.

- Akaryotes (Greek prefix 'a-' meaning "without"): Organisms without a nucleus or other membrane bound compartments.

The terms eukaryote and akaryote are comparative descriptions of cell ultrastructure. However, the term prokaryote is commonly used to represent organisms with akaryotic cell organization. The concept of a protein universe was put forward to organize proteins in a natural hierarchical system using methods of protein taxonomy (Ladunga, 1992). The number of distinct and stable 3D structures possible is limited by the physical and chemical constraints on protein folding, and, thus, the number of unique 3D structures (or Folds) possible was estimated to be finite. Furthermore, based on the relationship between sequence and structure divergence in proteins (Chothia \& Lesk, 1986), it was predicted that a vast majority of proteins belong to no more than a thousand structural families (Chothia, 1992). At the time of this prediction almost 30 
years ago, 866 structures were available in the PDB. In spite of the exponential growth of the number of structures available in PDB the prediction has turned out to be largely true. Structure-based protein taxonomy developed by SCOP (Murzin et al., 1995) and CATH (Orengo et al., 1997) classification systems identify $\approx 1,500$ and $\approx 1,400$ Folds, respectively.

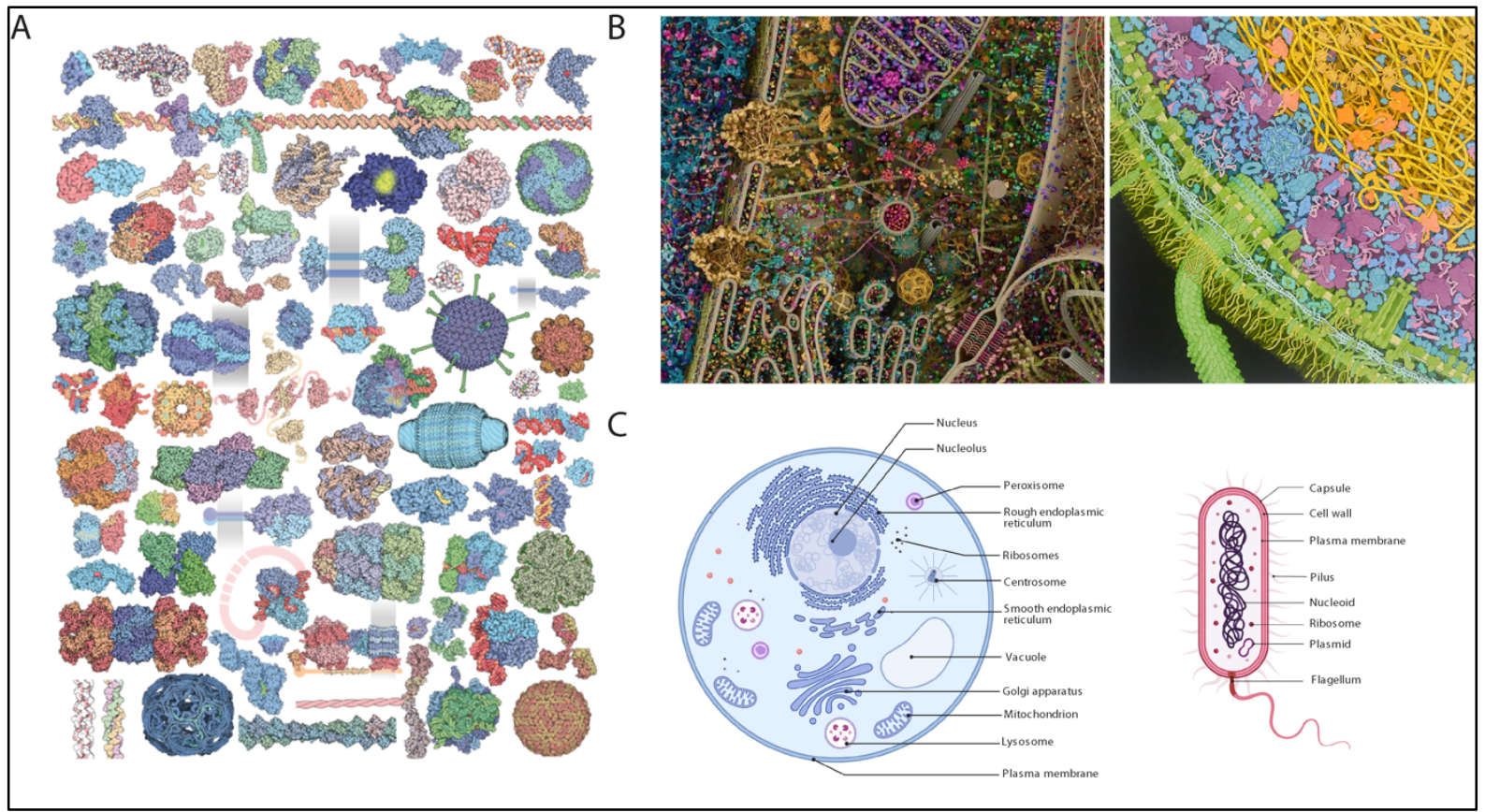

Figure 1. The molecular componentry of the cellular machinery. Most proteins fold into specific 3D shapes and form diverse complexes to perform their biological functions (A). In addition to carrying out the biochemical reactions, proteins build and maintain the morphological features of cells. Cells are membranous ensembles studded with proteins, inside and out. The two basic cell types - eukaryotic (nucleated) and akaryotic (anucleate) cells and the extent of membrane bound compartmentation are shown as section of the ultrastructure (B) and the overall structure of an average eukaryotic cell and an average akaryotic cell (C).

Despite being finite, and in spite of the remarkable advances in experimental 3D structure determination technologies, the protein universe is yet to be fully mapped (Levitt, 2009; Waman et al., 2020). Due to the relative ease of DNA sequencing, mapping genomes has far outpaced protein structure determination. Tools such as Alphafold and RoseTTaFold are a shot in the arm in the efforts to map the protein universe. At any rate, up to $70 \%$ of proteins of many cellular species can be mapped to known structures. This is already providing a substantial view of the distribution of proteomes in the cellular universe and the major patterns of diversification of cellular life (Figure 2).

In mapping the protein universe, a protein domain is the basic unit of structure, function and evolution (Figure 2A). Domains are independently folding sectors of a polypeptide chain, with a unique 3D shape that is associated with a distinct amino acid sequence profile and a characteristic function (Murzin et al., 1995; Orengo et al., 1997). For these reasons, domains are excellent "characters" to study many aspects of biological evolution. The character concept is central to evolutionary biology. A character is any recognizable and heritable trait, feature, or property of an organism (Figure 2A) that can be employed for comparative analysis of character variances as a measure of evolutionary divergence of species (Figure 2B). The 
idiosyncratic assortment of domains in organisms corresponds to species groups (Figure 2C) and other levels of the taxonomic hierarchy of organismal classification (Harish \& Kurland, 2017a; Harish et al., 2013). Therefore, tracing the history of the variation of domain composition in species is valuable for determining the evolutionary relationships and patterns of diversification among species groups. The species-specific composition of unique domains was termed as "intrinsic proteomic complexity" (Harish \& Kurland, 2017b).

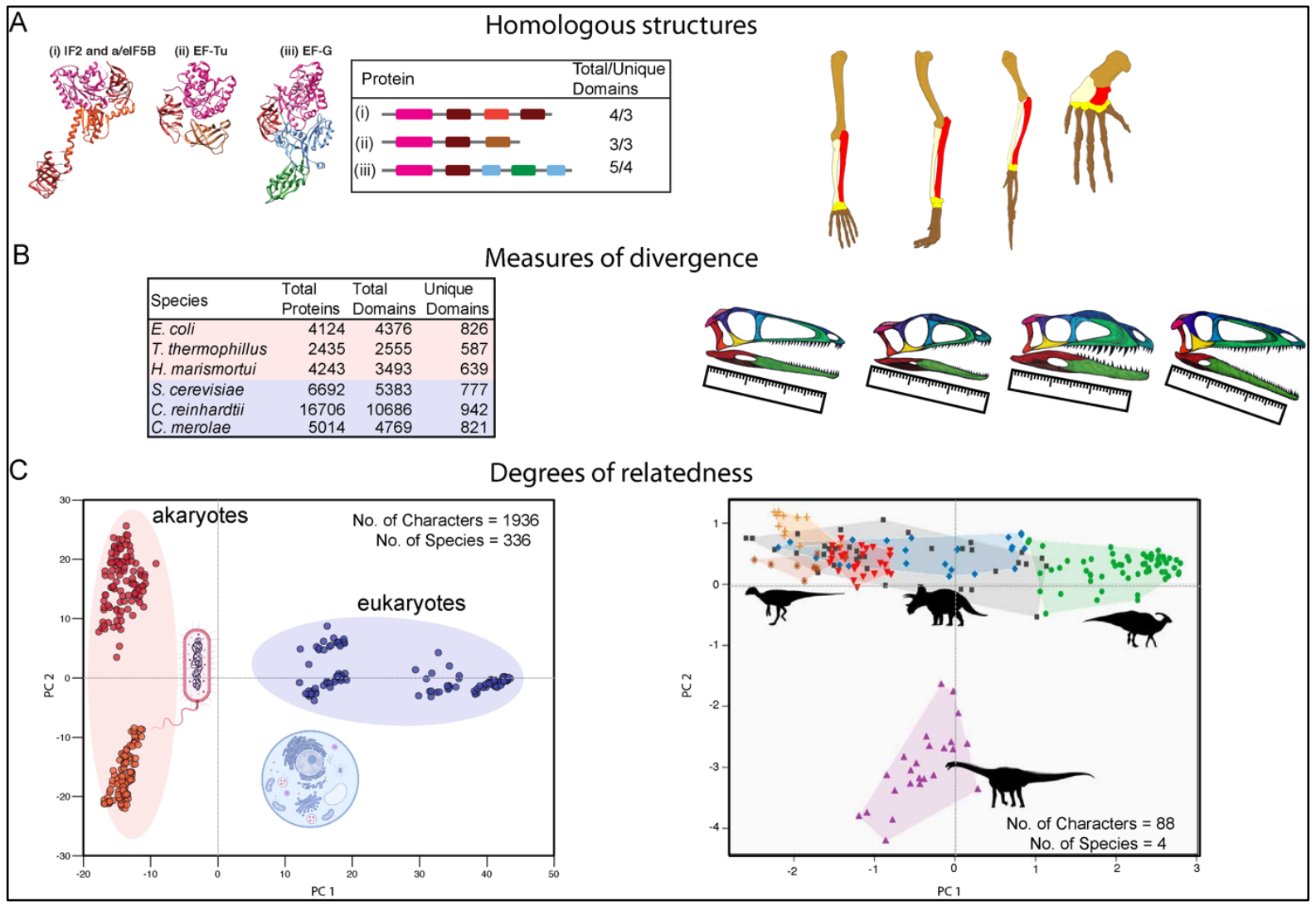

Figure 2. Protein domains are unique 'molecular phenotypes' to map patterns of species diversification. Structural domains are distinct homologous units that form complex protein morphological structures (A; left panel), comparable to complex morphological structures (A; right panel). Since domains are usually have characteristic functions, they are "functional genomic signatures" (Harish, 2018). Measures of compositional variation of domains is a good metric of divergence amongst organismal species groups (B; left panel). The number of unique occurrences defines a measure of "intrinsic proteomic complexity" (Harish \& Kurland, 2017a). Principal components analysis (PCA) projections show that the covariations of domain composition correspond to the two basic cell types (C; left panel). PC1 separates groups of eukaryote species from those of akaryote species while PC2 separates species groups within each of the eukaryotes and akaryotes. Domain-based metrics are comparable to measures of variance in fossil jaw and dental homologs (B; right panel), and the covariations of these features correspond to clades of dinosaur species (C; right panel taken from (Nordén et al., 2018)).

Contemporary species are the evolutionary successors of long-gone ancestors. About $99 \%$ of species that evolved on Earth have gone extinct (Barnosky et al., 2011) with little trace left as fossils, especially of microbial species. Therefore, reconstructing a detailed picture of the common ancestor of all extant life - the universal common ancestor - is a daunting task (Harish \& Morrison, 2020). The universal common ancestor (UCA) was most likely a singlecelled species estimated to have lived between 3.8 to 3.5 billion years ago (BYa). Otherwise, the nature of UCA is still fuzzy and rife with speculation. When it comes to peering back into 
the distant past, astrophysicists and evolutionary biologists are faced with similar problems in collecting reliable data and building tools to analyze and interpret the data (Kurland \& Harish, 2015).

\section{Farsightedness and nearsightedness}

Studies to reconstruct the cosmological past initially relied on refracting telescopes. Telescopes are the fundamental tools to study the observable universe. Refracting telescopes are one of the two main types of optical telescopes, which operate by collecting light through a large lens and focusing the light on an eyepiece/camera (STScI, 2021). However, refracting telescopes suffer from a phenomenon called chromatic distortion - a common optical problem resulting from an inability of the lens to bring all wavelengths of light to a sharp focus. As a result, the reconstructed images of distant galaxies - galactic ancestors of contemporary universe, are fuzzy. In a telescope, the function of a good lens is to minimize such optical aberrations as much as possible to produce an unblurred and high-fidelity view. The deficiencies of refracting telescopes were overcome by reflecting telescopes wherein the lens was replaced with a mirror to collect light and focus better for a clearer picture. The bigger the mirror the better. The Hubble and Webb space telescopes are the largest reflecting telescopes that can collect high quality data of the most distant 'galactic fossils' of the cosmological universe.

The observable cosmic universe converges into a singularity known as the cosmological light horizon, which represents beginnings of the universe and the boundary between the observable and unobservable universe (Figure 3A). In reconstructing the biological past, the universal common ancestor (UCA), represents a singularity - a phylogenetic event horizon (Figure 3B) - which is the root node of the universal tree of life (Harish et al., 2013). Among the tools used to peer back into the galaxies of the cellular universe, protein structure-based evolutionary telescopes are like the reflecting telescopes with minimal distortions (Harish, 2018; Harish \& Kurland, 2017a). Therefore, structure-based telescopes produce a well-resolved and highfidelity picture of the distant biological past (Figure 3B). In contrast, their predecessors sequence-based telescopes produce a poorly-resolved and low-fidelity picture of the past, which makes the identity and nature of UCA ambiguous (Figure 3C).

Resolving the deeper nodes of the universal tree of life (hereafter universal tree) in general, and locating the root node in particular using sequence-telescopes is fraught with distortions similar to the chromatic aberrations of refracting telescopes (Harish, 2018). This is because (a) the rates of substitution mutations in sequences show extreme variations, unlike the speed of light, which is a physical constant, and (2) the historical signal decays significantly with time due to repeated substitutions that overwrite the evolutionary record (Harish \& Kurland, 2017a; Harish \& Morrison, 2020). The decay of historical signal is pronounced in primary sequences of genes/proteins, which increases spurious signals and decreases the reliability of analyses. While distortions due rate variations can be corrected with mathematical models, decay and loss of signal cannot be compensated (Harish, 2018). Thus, distortions of evolutionary signal and high uncertainty in identifying the UCA is common with comparative analysis of primary sequence data (Harish, 2018; Harish \& Morrison, 2020; Liu et al., 2021; Williams et al., 2020). 


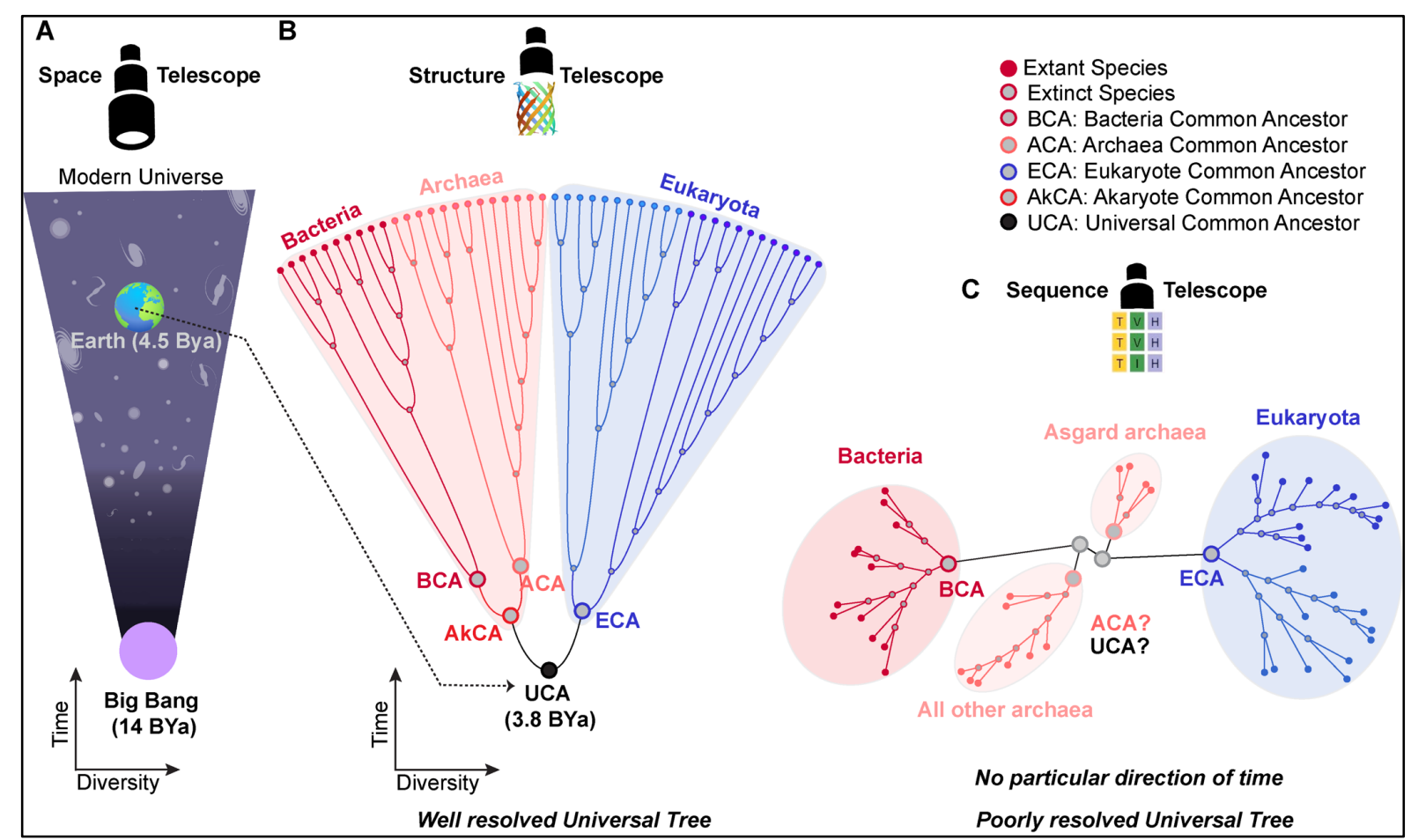

Figure 3. Different types of telescopes used to peer into the depths of the cosmological and cellular universe. Optical telescopes are used to look at the distant 'galactic fossils' of the cosmological universe estimated to have originated $\approx 14$ billion years ago (BYa) (A). Phylogenies are the "evolutionary telescopes" used to look back into the distant biological past depicted as the "universal tree" of life. Protein structure-telescopes can look further back in time due to their superior resolving power (B) compared to the commonly used sequence-telescopes (C). The root node or the UCA of the universal tree cannot be identified using sequence-telescopes, hence the reconstructed picture of evolution is poorly resolved and incomplete $(\mathrm{C})$.

\section{Nothing in the universal tree makes sense except in the light of the universal ancestor}

During last two decades the universal tree is routinely constructed as a composite tree of 3050 phylogenetic "marker" proteins/genes (Ciccarelli et al., 2006; Hug et al., 2016; Liu et al., 2021), rather than from a single marker gene: ribosomal RNA (rRNA) gene (Woese, 1987). Marker protein datasets are either solely, or predominantly composed of ribosomal proteins (Harish, 2018). Sequence-based analyses trace the history of substitution mutations in primary sequences. Time-reversible substitution models are widely used in phylogenetic analysis. However, time-reversible models infer "unrooted trees" and hence lack the ability to identify the root node, which in the case of the universal tree is represents the UCA. This inherent deficiency of the sequence-telescopes was noted early on (Woese, 1987). Because of the total absence of a root node, the deficiency of widely used sequence-telescopes is, in fact, worse than chromatic distortion of refracting telescopes (Harish, 2018; Harish \& Morrison, 2020). An unrooted universal tree (Figure $3 \mathrm{C}$ ) is therefore not only poorly resolved but is also an incomplete depiction of evolution.

In general, a "rooted tree" is a straightforward depiction of the principle of common ancestry with a clear branching order along a time axis of ancestor-descendant polarity (Figure 3B). In contrast, an unrooted tree is undirected with no particular direction for evolutionary time, and thus, with undefinable branching order (Figure 3C). This distinction between an unrooted and a rooted tree is highly significant as most conclusions from phylogenetic analyses strictly 
depend on a rooted tree. The primary conclusions of significance include determining (a) ancestors and descendants; (b) branching order (i.e., tree topology); (c) evolutionary groups (clades); and (d) degree of relatedness among clades. Hence, unrooted trees are not evolutionary trees (phylogenies) in a strict sense, even though they depict relatedness among the organisms.

Evidently, the importance of identifying the UCA cannot be emphasized enough (Harish \& Kurland, 2017b). Yet, rooting is relegated as a secondary task and is often trivialized. As commonly used phylogenetic routines cannot identify the root, "pseudo rooting" (see Box 1) based on external information that is independent of the data used to produce unrooted trees becomes necessary. Pseudo-rooting converts undirected trees to directed trees so that evolutionarily meaningful conclusions can be drawn (Harish, 2018; Woese, 1987). Depending on the external information (e.g., outgroups) or theoretical assumptions about the UCA, pseudo-rooting of the universal tree is the common practice (Imachi et al., 2020; Liu et al., 2021; Spang et al., 2015; Williams et al., 2020; Woese et al., 1990). The assumptions underpinning pseudo-rootings of the universal tree are widely accepted but are rarely tested (Harish, 2018; Harish \& Kurland, 2017a).

The difficulty of locating the root node in sequence-based analysis, and the importance of a statistically robust root inference were recently highlighted in efforts to trace the origin of (a) the human severe acute respiratory syndrome coronavirus 2 (SARS-CoV-2), and (b) the coronavirus 2019 (COVID-19) pandemic (Morel et al., 2021; Pipes et al., 2021). One study claimed to have identified the ancestors of the human SARS-CoV-2 lineages (Forster et al., 2020) by including the bat corona virus as outgroup to root a median joining network (MJN). Rather than tracing the history of substitution mutations, MJNs estimate genetic distances, which is inappropriate to trace evolutionary history of mutations and to reconstruct ancestral sequence states (Sánchez-Pacheco et al., 2020).

In contrast, several other studies employed appropriate substitution models and more rigorous statistical phylogenetic methods to evaluate multiple rootings (Morel et al., 2021; Pipes et al., 2021). The SARS-CoV-2 tree was (pseudo)rooted using the outgroup criterion with multiple outgroups or rooted with molecular clock models and non-reversible substitution models. Yet, an unambiguous and statistically robust rooting was not possible using the best available methods of primary sequence analysis, and, in spite of the availability of massive wholegenome datasets. The difficulty and unreliability of rooting the SARS-CoV-2 tree was due to a rapid loss of evolutionary signal (Morel et al., 2021; Pipes et al., 2021). Poor resolution due to lack of evolutionary signal along with misinterpretation of unrooted trees can lead to profoundly misleading conclusions in many other evolutionary studies as well (Baum et al., 2005; Graham et al., 2002; Harish, 2018). The shortcomings of unrooted MJNs and potential misinterpretations were pointed out in a sharp response as "Median-joining network analysis of SARS-CoV-2 genomes is neither phylogenetic nor evolutionary" (Sánchez-Pacheco et al., 2020). 


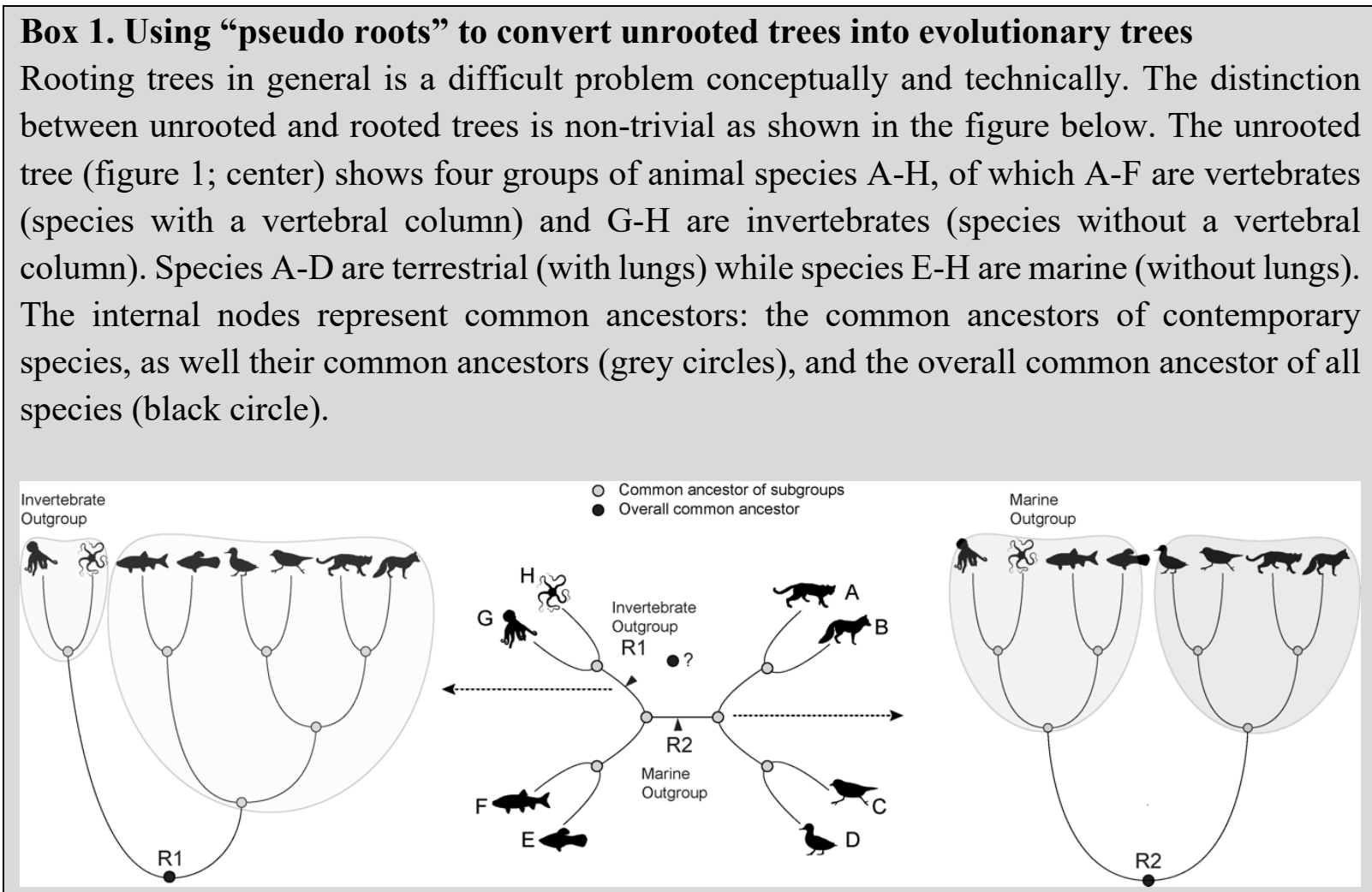

Box 1-figure 1. Examples of different rearrangements of the branching order following an outgroup rooting. Depending on the different positions of the root node, the different degrees of relatedness amongst species groups can be inferred. The closest relative in an unrooted tree may not be the closest evolutionary relative. The degree of relatedness can only be determined with rooted trees. Trees are drawn as cladograms with emphasis on branching order and relative age of common ancestors of contemporary species, branch lengths have evolutionary implications.

Commonly used phylogenetic routines produce "unrooted trees", in which (a) the position of the overall common ancestor cannot be identified, and (b) nor a particular direction for evolutionary time is implied. Hence unrooted trees are incomplete depictions of evolution (figure 1; center). To complete the picture, and outgroup can be chosen to assign a "pseudo root". The addition of the root node introduces a branching order by rearranging the tree around the root node (figure 1 left and right). Outgroups can be chosen based on assumptions about features (i.e., characters). For e.g., either presence or absence of lungs/vertebrae is assumed to be the ancestral state. Since an artificial root node is introduced after-the-fact, it is designated as a "pseudo root".

Fossil evidence confirms that the invertebrate outgroup assumption is correct as far as the Animal tree is concerned. As for the Universal tree, in the absence of both organismal outgroups and reliable geological fossils, protein domains are perhaps the best characters available at present (Harish, 2018). Locating the root node (UCA) with a directional characterevolution model is the most straightforward rooting approach. Accordingly, empirical evidence in favor of the descent of eukaryotes and akaryotes from UCA supports Eukaryota and Akaryota as the primary clades of life (see Figure 4 and associated discussion). 
Likewise, significant loss of evolutionary signal in routinely used primary sequence data is the basis of the problems and persistent ambiguities in resolving the deeper nodes of the universal tree (Harish, 2018). Thanks to structure-telescopes, the deficiencies of sequence-telescopes can now be overcome so that a well-supported and well-resolved universal tree can be reconstructed. The advantages of embracing structure-based characters for studying evolution are manifold (see next section). However, the routine use of sequence characters with low evolutionary signal goes hand in hand with the common practice of pseudo-rooting (Lake, 1986; Liu et al., 2021; Woese et al., 1990).

Pseudo-rootings are routinely used to assert that (a) archaea are the closest relatives of eukaryotes (Woese et al., 1990); (b) eukaryotes evolved from a specific lineage of archaea (Imachi et al., 2020; Spang et al., 2015); and (c) archaea are intermediates on the evolutionary path to eukaryotes from bacteria (Imachi et al., 2020). However, un-verified pseudo-rootings may be faulty, and the practice of pseudo-rooting the universal tree solely for interpreting the results of an unrooted analysis can therefore lead to false results. Indeed, unsupported falserootings along with poorly resolved trees tend to foster common misconceptions about evolution (Harish et al., 2016; Harish \& Kurland, 2017b; Sánchez-Pacheco et al., 2020).

Misreading of even well resolved, rooted trees is surprisingly common (Baum et al., 2005). A likely reason is that evolutionary trees are often read along the tips (terminal nodes) of the tree, which represents extant organisms, rather than along the path from the root node to the tips through the internal nodes, which is the line of descent from common ancestors. For instance, the universal tree in which eukaryotes and akaryotes descend and diverge from the UCA (Figure 3B) is often misinterpreted as a 'eukaryotes first' scenario or an 'upside down' tree of life since it contradicts the common false-rooting that assumes (a) UCA is a primitive prokaryote, and (b) prokaryote-to-eukaryote progression (Harish \& Kurland, 2017a; Harish \& Morrison, 2020). Rather, the straightforward conclusion is that eukaryotes and akaryotes are sister clades, and that the closest relative of the eukaryote common ancestor (ECA) as well as the akaryote common ancestor (AkCA) is UCA (Figure 3B).

\section{Roots of stability: The diminishing relevance of the three domains classification system}

The Linnean system of organizing life into nested hierarchies Systema Naturae (Linnaeus, 1758) was developed a century before Darwin's oft-cited vision "Our classifications will come to be, as far as they can be so made, genealogies" (Darwin, 1859). The term phylogeny was coined when one of the first genealogical tree of life was depicted (Haeckel, 1866), inspired by the principle that a "natural system" and a true classification should be represented as a genealogical tree (Darwin, 1859). Yet, some of the prominent genealogical trees (Figure 4AC) are not Darwinian trees. In Darwinian phylogenetic trees, contemporary species are at the leaves (terminal nodes) and extinct ancestors at the internal nodes. Many notable hypotheses of phylogenetic progression assume, explicitly or implicitly, that some extant species groups are primitive (Figure 4, A-C), much like the popular depictions of evolution as a linear progression from simple to complex forms. For instance, unicellular species with akaryotic cell organization were assumed to be primitive and placed near 'virtual root' of the tree (Figure 4A; (Haeckel, 1866)), (Figure 4B; (Whittaker, 1969)) and (Figure 4C; (Woese, 1987)). However, 
the venerable ancestor - UCA in this case, was neither a distinct entity nor an empirically derived node on the phylogeny. Hence, neither the three-kingdom system (Figure 4C; (Woese, 1987)) nor its predecessor, the five-kingdom system (Figure 4B; (Whittaker, 1969)) are strictly phylogenetic.

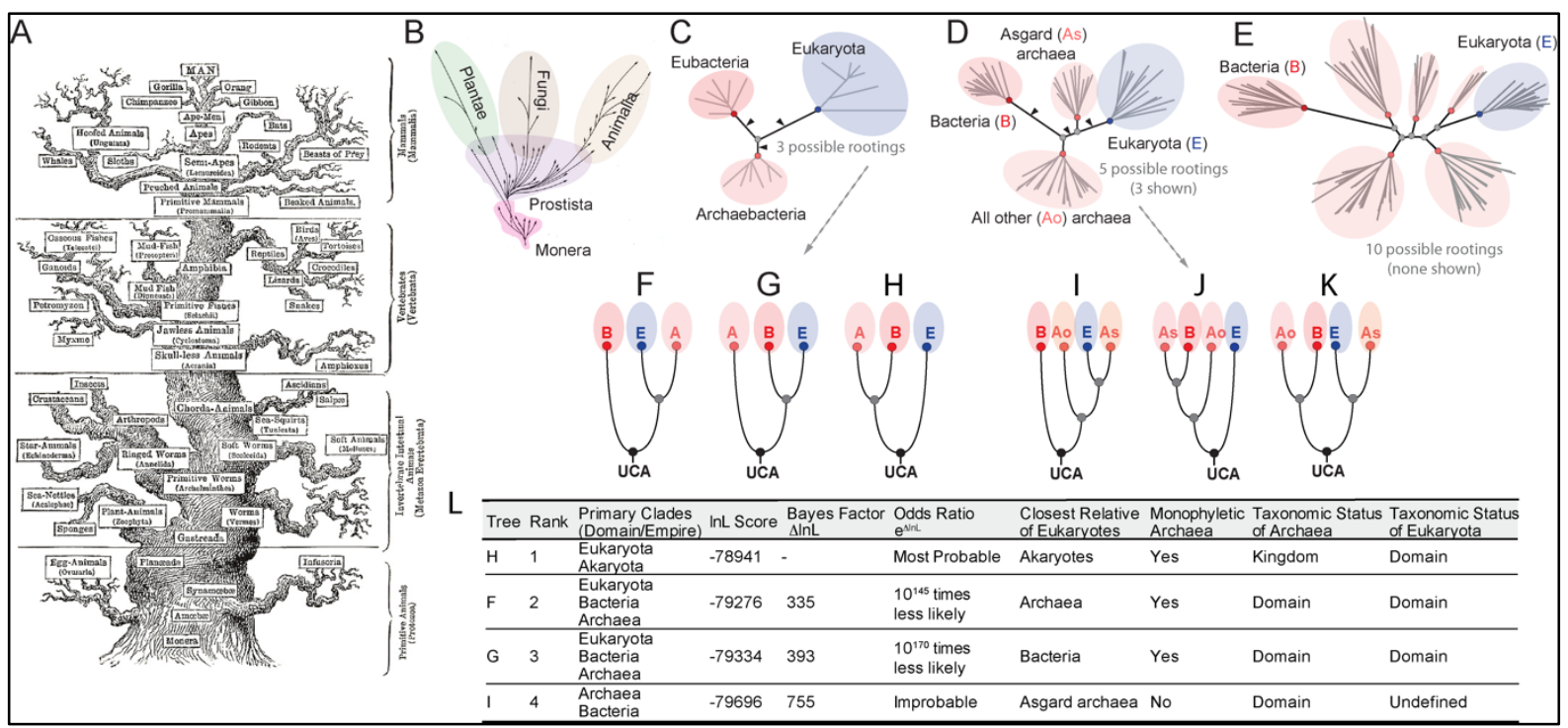

Figure 4. Assessment of empirical evidence for or against alternative universal trees and for identifying the primary clades of life. The identification of the primary clades in the universal tree is basically linked to the identity of the root node (UCA), which is implicitly assumed ever since the earliest universal trees were put forward (A-E). The most popular assumption (Woese et al., 1990) is that UCA is positioned on the stem-branch leading to $(\mathrm{Eu})$ Bacteria $(\mathrm{C})$ and the text book universal tree $(\mathrm{F})$. Several other phylogenetic positions for the UCA and the resulting phylogenies (D, G-K). Assessment of empirical evidence for or against these proposals (L) shows that the universal tree in which Eukaryota and Akaryota are the primary clades $(\mathrm{H})$ is most likely to be correct.

The poor resolution of archaea due to unreliable evolutionary signal in routinely used 'marker sequences' is often seen as non-monophyly of archaea (Figure 4D-E). Regardless of rooting, poor resolution of archaea further confounds phylogenetic classification (Figure 4I-K). If we are to accept a poorly resolved universal tree, then some of the possibilities depending on different rootings are,

- Eukaryota ceases to exist as an exclusive clade and a taxonomic Domain (Figure 4I).

- Bacteria ceases to exist as an exclusive clade and a taxonomic Domain (Figure 4J)

- All of Eukaryota, Bacteria and Archaea cease to exist as exclusive clades or Domains of life (Figure 4K)

Arguably, resolving the root node of the universal tree is one of the hardest problems in evolutionary biology given the time-depth (Harish, 2018). Fortunately, structural characters cut the Gordian knot by facilitating an empirical resolution of the rooting problem as well as diagnosis of the monophyly of the major species groups by allowing the quantification of empirical evidence in favor of the different suppositions and tentative hypotheses (Figure 4L). Bayes Factors (BF) provide a means to evaluate the strength of evidence in favor of the best hypothesis among a set of competing proposals (Harish, 2018; Harish \& Kurland, 2017a). BF is the ratio of the likelihoods of the different hypotheses being compared. A BF of 5 or greater is considered as very strong empirical evidence in favor of the hypothesis with the better 
likelihood. Therefore, a BF of 335 means extremely strong empirical evidence for the twodomains universal tree (Figure $4 \mathrm{H}$ ) and for primary clades Eukaryota and Akaryota (Bacteria and Archaea are sister clades), compared to other competing hypotheses (Harish, 2018). Likelihood scores are the $\log$ odds of the hypotheses, thus the Eukaryota-Akaryota twodomains phylogeny is at least $10^{145}$ times more probable than the closest competing phylogeny (Figure 4F), which is the three-domains phylogeny (Eukaryota and Archaea are sister clades). The alternative two-domains proposal and non-monophyly of Archaea are improbable and least supported by empirical evidence. Put simply, the Eukaryota-Akaryota two-domains phylogeny is most likely to be correct.

The universal tree is primarily a phylogenetic classification. The basic requirements of phylogenetic classification are,

(a) Monophyly: Only monophyletic groups (or clades) are true evolutionary groups. That is, groups comprising all the descendants of a given common ancestor should be identified.

(b) Homology: Delineating clades is based on diagnosing patterns of descent of characters that evolved in the common ancestor and were inherited by the descendants (i.e., homologous characters). Nested configurations of sharing of such homologous characters in different species are used to group them in an order of common descent (i.e., branching order), which then diagnoses the degree of relatedness among the clades.

This seemingly straightforward procedure of character analysis and the algorithmic logic to diagnose clades was developed so that phylogenetic classification is an objective exercise determined by the branching order (Hennig, 1965).

However, the common practice of pseudo-rooting the universal tree is essentially based on an unverified assumption and encourages a subjective interpretation of unrooted trees. Though appealing and widely accepted, the assumption was rarely tested until recently (Harish, 2018; Harish \& Kurland, 2017a). Perhaps because it was not feasible with sequence-characters, or because the pseudo-rooting aligns with another common traditional assumption: that simple is primitive (Harish \& Kurland, 2017a). This assumption is pervasive since the time of the earliest efforts to reconstruct a genealogical tree of life (Figure 4A-C). The current practice of pseudorooting the universal tree (Liu et al., 2021; Zaremba-Niedzwiedzka et al., 2017) goes back to the initial efforts of classification of life using molecular characters (Woese et al., 1990). Thus, conclusions based on pseudo-rooting are compromised both by a lack empirical evidence and their reliance on poorly quality data (Harish, 2018). Notably, the widely accepted, but rarely tested notion that eukaryotes evolved from prokaryotes is most seriously compromised due to the strong evidence for the monophyly of archaea and because the routinely used false-rootings and the underlying assumptions lack support.

Advantages of protein domains for assessing both qualitative and quantitative empirical evidence are summarized below (for technical details of implementation and performance of 
different methods/models see (Harish, 2018; Harish \& Kurland, 2017a, 2017b; Harish \& Morrison, 2020).

- Protein domains, unlike their component amino acids provide for a large number of "unique" characters. Latest updates of SCOP and CATH classification of PDB entries identify $\approx 2,750$ and $\approx 5,500$ homologous superfamilies. This translates to anywhere between 2,750 and 5,500 unique structure-characters as opposed to only 20 distinct sequence-characters (amino acids).

- Since each homologous domain has a distinct 3D structure, a unique sequence profile and a characteristic function, substitution between domains is not known. In contrast, repeated substitution of amino acids at the same site is frequent, resulting in a rapid decay of historical signal.

- Independent evolution of complex structural domains in diversified species and $a b$ initio evolution of new proteins by random mutations are both extremely rare. However, it is relatively easier to lose domains via multiple mechanisms. For example, a mutation causing a premature stop codon or loss of a genomic locus during genetic recombination. This naturally skewed propensity for loss (death) over gain (birth) of a new domain can be exploited to implement non-reversible or directional-evolution models, which are better suited to reconstruct the universal tree.

- Finally, the relatively lower variation of (a) compositional heterogeneity, and (b) rate heterogeneity of birth/death of unique domains compared to point mutations in sequences, supports statistically robust phylogenetic inferences.

Thus, structure-telescopes provide for (a) a robust and reliable resolution of the deeper nodes of the universal tree. However, the shallow parts of the tree are not as well supported as the deeper ones when only structure characters are used (Harish, 2018). Combining sequence and structural characters could improve the resolution of all parts of the universal tree.

\section{Conclusions}

Structure-telescopes provide for a straightforward and objective means to identify the UCA and to determine the universal phylogeny. Protein structural domains define unique molecular phenotypes, which are robust evolutionary characters that improve the level of confidence in resolving the deeper divergences of the universal tree, including the root node. It is abundantly evident that, in addition to a richer representation of cellular phenotypes, protein domains offer a deeper perspective of the evolutionary history in sharp contrast to the widely used sequencecharacters. Thus, they provide for a better means of (a) resolving the deeper nodes of the universal tree, (b) objective evaluation of competing hypotheses for early evolution of cellular life, and (c) phylogenetic classification, which is in an accurate representation of the two basic types of cell organization.

The universal phylogeny wherein eukaryotes and akaryotes are sister clades is by far the best empirically supported universal tree of life by any measure, qualitative and quantitative. To elaborate, qualitative measures include both the tree-based assessment of common ancestrycharacter homology and clade homology, and tree-independent assessment of character homology. Quantitative measures include robust statistical support for (a) best-fitting character 
evolution model (b) the branching order, starting with the rooting, and (c) higher confidence measures against alternative universal tree proposals. Hence structure-telescopes are better suited in contrast to sequence-telescopes to look further back into the biological past. The serious limitation of sequence characters, especially with regard to the assessment of qualitative evidence can be overcome with structure characters.

Perhaps, portraying the pros and cons of the sequence-based and structure-based reconstruction of the universal tree as a 'battle of characters' would make for an entertaining tale. However, both types of molecular features are complementary and are valuable for resolving different parts of the universal tree. That is, by melding together structure-telescopes and sequencetelescopes, both farsightedness and nearsightedness of evolutionary telescopes can be corrected. During the last two decades, neither increasing the sophistication of the substitution models nor aggregating more sequences, has been productive in reliably resolving deeper divergences of the universal tree. Embracing the well-defined structure-based characters will certainly prove to be beneficial.

The durability of Linnean classification rests on the choice of excellent 'diagnostic features' or characters used to group species into genera, families through Kingdoms. If only high-quality molecular characters such as protein domains were available early on, perhaps there never would have been a third domain of life. Hindsight is 20/20, after all. The Linnean hierarchical classification implicitly reflected common descent of the species thus classified, and ultimately converged into an Empire, Imperium Naturae. Although the taxonomic grade Domain/Empire is warranted, in light of the new, stable rooting and well-supported branching order of the universal tree, grades for Archaea and Bacteria should be revised to Kingdoms, whether or not their respective initial nomenclatures Archaeabacteria and Eubacteria should take precedence. Eukaryota and Akaryota are the two primary Domains of Life, both terms being descriptive of the two basic cell types.

As genome sequences and protein structures continue to accumulate, future efforts for a better resolved universal tree could employ a variety of new molecular features. In addition to primary sequences and known protein domains, many newer evolutionary characters can be identified by determining (a) novel protein domains for which structures are unknown; and (b) new types of homologous features from quaternary assemblies of the protein complexes. Tools like AlphaFold and RoseTTaFold seem to be primed for such undertakings. In this way, the evolution of morphological phenotypes as well as physiological phenotypes at the cellular level can be reconstructed in greater detail than what is possible at present.

\section{Data availability}

No new data were generated in this study.

\section{Image credits}

Figure 1A: Molecule of the Month, Illustration by David S. Goodsell, RCSB Protein Data Bank Figure 1B, LHS: Eukaryotic cellular landscape, Illustration by Evan Ingersoll \& Gaël McGill, Ph.D. (Digizyme Inc.) using Molecular Maya software. Created for Cell Signaling Technology, 
Inc., and inspired by the stunning art of David Goodsell, this 3D rendering of a eukaryotic cell is modeled using X-ray, nuclear magnetic resonance (NMR), and cryo-electron microscopy datasets for all of its molecular actors.

Figure 1B, RHS: Escherichia coli Bacterium, 2021, Illustration by David S. Goodsell, RCSB Protein Data Bank. doi: 10.2210/rcsb_pdb/goodsell-gallery-028

Figure 1C: Created with BioRender.com

Figure 2A, RHS: Volkov Vladislav Petrovich, Wikimedia Commons, CC BY-SA 4.0

Figure 2B, RHS: Danny Cicchetti, Wikimedia Commons, CC BY-SA 4.0

Figure 3A: First Stars: Timeline of the Universe, Space Telescope Science Institute (STSci).

Figure 4A: Tree of life, by Ernst Haeckel, Wikipedia.org

Box 1-figure 1: Silhouette images of animals, by PhyloPic database.

\section{References}

Baek, M., DiMaio, F., Anishchenko, I., Dauparas, J., Ovchinnikov, S., Lee, G. R., Wang, J., Cong, Q., Kinch, L. N., Schaeffer, R. D., Millán, C., Park, H., Adams, C., Glassman, C. R., DeGiovanni, A., Pereira, J. H., Rodrigues, A. V., van Dijk, A. A., Ebrecht, A. C., Opperman, D. J., Sagmeister, T., Buhlheller, C., Pavkov-Keller, T., Rathinaswamy, M. K., Dalwadi, U., Yip, C. K., Burke, J. E., Garcia, K. C., Grishin, N. V., Adams, P. D., Read, R. J., \& Baker, D. (2021). Accurate prediction of protein structures and interactions using a three-track neural network. Science, eabj8754. https://doi.org/10.1126/science.abj8754

Bank, P. D. (1971). Crystallography: Protein Data Bank. Nat. New Biol., 233(42), 223.

Barnosky, A. D., Matzke, N., Tomiya, S., Wogan, G. O., Swartz, B., Quental, T. B., Marshall, C., McGuire, J. L., Lindsey, E. L., \& Maguire, K. C. (2011). Has the Earth's sixth mass extinction already arrived? Nature, 471(7336), 51-57.

Baum, D. A., Smith, S. D., \& Donovan, S. S. S. (2005). The tree-thinking challenge. Science, 310(5750), 979-980.

Chothia, C. (1992). One thousand families for the molecular biologist. Nature, 357(6379), $543-$ 544.

Chothia, C., \& Lesk, A. M. (1986). The relation between the divergence of sequence and structure in proteins. The EMBO journal, 5(4), 823-826.

Ciccarelli, F. D., Doerks, T., von Mering, C., Creevey, C. J., Snel, B., \& Bork, P. (2006). Toward Automatic Reconstruction of a Highly Resolved Tree of Life. Science, 311(5765), 12831287. https://doi.org/10.1126/science.1123061

Darwin, C. (1859). On the Origin of Species by Means of Natural Selection, or the Preservation of Favoured Races in the Struggle for Life. John Murray.

Forster, P., Forster, L., Renfrew, C., \& Forster, M. (2020). Phylogenetic network analysis of SARS-CoV-2 genomes. Proceedings of the National Academy of Sciences, 117(17), 9241-9243.

Gierasch, L. M., \& Berman, H. M. (2021). How the Protein Data Bank changed biology: An introduction to the JBC Reviews thematic series, part 2. Journal of Biological Chemistry, 296.

Graham, S. W., Olmstead, R. G., \& Barrett, S. C. H. (2002). Rooting Phylogenetic Trees with Distant Outgroups: A Case Study from the Commelinoid Monocots. Molecular biology and evolution, 19(10), 1769-1781. 
Haeckel, E. (1866). Generelle Morphologie der Organismen: Bd. Allgemeine Entwickelungsgeschichte der Organismen (Vol. 2). G. Reimer.

Harish, A. (2018). What is an archaeon and are the Archaea really unique? PeerJ, 6, e5770. https://doi.org/10.7717/peerj.5770

Harish, A., Abroi, A., Gough, J., \& Kurland, C. (2016). Did viruses evolve as a distinct supergroup from common ancestors of cells? Genome Biology and Evolution, 8(8), 2474-2481.

Harish, A., \& Kurland, C. G. (2017a). Akaryotes and Eukaryotes are independent descendants of a universal common ancestor. Biochimie, 138, 168-183.

Harish, A., \& Kurland, C. G. (2017b). Empirical genome evolution models root the tree of life. Biochimie, 138, 137-155.

Harish, A., \& Morrison, D. (2020). The deep(er) roots of Eukaryotes and Akaryotes [version 2; peer review: 2 approved, 1 approved with reservations]. F1000Research, 9(112). https://doi.org/10.12688/f1000research.22338.2

Harish, A., Tunlid, A., \& Kurland, C. G. (2013). Rooted phylogeny of the three superkingdoms. Biochimie, 95(8), 1593-1604.

Hennig, W. (1965). Phylogenetic systematics. Annual review of entomology, 10(1), 97-116.

Hug, L. A., Baker, B. J., Anantharaman, K., Brown, C. T., Probst, A. J., Castelle, C. J., Butterfield, C. N., Hernsdorf, A. W., Amano, Y., \& Ise, K. (2016). A new view of the tree of life. Nature Microbiology, 1, 16048.

Imachi, H., Nobu, M. K., Nakahara, N., Morono, Y., Ogawara, M., Takaki, Y., Takano, Y., Uematsu, K., Ikuta, T., Ito, M., Matsui, Y., Miyazaki, M., Murata, K., Saito, Y., Sakai, S., Song, C., Tasumi, E., Yamanaka, Y., Yamaguchi, T., Kamagata, Y., Tamaki, H., \& Takai, K. (2020). Isolation of an archaeon at the prokaryote-eukaryote interface. Nature, 577(7791), 519-525. https://doi.org/10.1038/s41586-019-1916-6

Kurland, C. G., \& Harish, A. (2015). The phylogenomics of protein structures: The backstory. Biochimie, 119 ,

284-302. https://doi.org/http://dx.doi.org/10.1016/j.biochi.2015.07.027

Ladunga, I. (1992). Phylogenetic continuum indicates "galaxies" in the protein universe: preliminary results on the natural group structures of proteins. Journal of Molecular Evolution, 34(4), 358-375.

Lake, J. A. (1986). An alternative to archaebacterial dogma. Nature, 319(6055), 626-626.

Levitt, M. (2009). Nature of the protein universe. Proceedings of the National Academy of Sciences of the United States of America, 106(27), 11079-11084.

Linnaeus, C. v. (1758). Systema naturae, vol. 1. Systema naturae, Vol. 1.

Liu, Y., Makarova, K. S., Huang, W.-C., Wolf, Y. I., Nikolskaya, A. N., Zhang, X., Cai, M., Zhang, C.-J., Xu, W., \& Luo, Z. (2021). Expanded diversity of Asgard archaea and their relationships with eukaryotes. Nature, 593(7860), 553-557.

Locey, K. J., \& Lennon, J. T. (2016). Scaling laws predict global microbial diversity. Proceedings of the National Academy of Sciences, 113(21), 5970-5975.

Morel, B., Barbera, P., Czech, L., Bettisworth, B., Hübner, L., Lutteropp, S., Serdari, D., Kostaki, E.-G., Mamais, I., \& Kozlov, A. M. (2021). Phylogenetic analysis of SARS-CoV-2 data is difficult. Molecular biology and evolution, 38(5), 1777-1791.

Murzin, A. G., Brenner, S. E., Hubbard, T., \& Chothia, C. (1995). SCOP: A structural classification of proteins database for the investigation of sequences and structures. Journal of Molecular Biology, 247(4), 536-540. 
Nordén, K. K., Stubbs, T. L., Prieto-Márquez, A., \& Benton, M. J. (2018). Multifaceted disparity approach reveals dinosaur herbivory flourished before the end-Cretaceous mass extinction. Paleobiology, 44(4), 620-637.

Orengo, C. A., Michie, A. D., Jones, S., Jones, D. T., Swindells, M. B., \& Thornton, J. M. (1997). CATH - A hierarchic classification of protein domain structures. Structure, 5(8), 10931108.

Pipes, L., Wang, H., Huelsenbeck, J. P., \& Nielsen, R. (2021). Assessing uncertainty in the rooting of the SARS-CoV-2 phylogeny. Molecular biology and evolution, 38(4), 15371543.

Sánchez-Pacheco, S. J., Kong, S., Pulido-Santacruz, P., Murphy, R. W., \& Kubatko, L. (2020). Median-joining network analysis of SARS-CoV-2 genomes is neither phylogenetic nor evolutionary. Proceedings of the National Academy of Sciences, 117(23), 1251812519.

Spang, A., Saw, J. H., Jørgensen, S. L., Zaremba-Niedzwiedzka, K., Martijn, J., Lind, A. E., van Eijk, R., Schleper, C., Guy, L., \& Ettema, T. J. (2015). Complex archaea that bridge the gap between prokaryotes and eukaryotes. Nature, 521(7551), 173-179.

STScl. (2021). Webb Telescope. https://www.stsci.edu/communications-and-outreach

Tunyasuvunakool, K., Adler, J., Wu, Z., Green, T., Zielinski, M., Žídek, A., Bridgland, A., Cowie, A., Meyer, C., Laydon, A., Velankar, S., Kleywegt, G. J., Bateman, A., Evans, R., Pritzel, A., Figurnov, M., Ronneberger, O., Bates, R., Kohl, S. A. A., Potapenko, A., Ballard, A. J., Romera-Paredes, B., Nikolov, S., Jain, R., Clancy, E., Reiman, D., Petersen, S., Senior, A. W., Kavukcuoglu, K., Birney, E., Kohli, P., Jumper, J., \& Hassabis, D. (2021). Highly accurate protein structure prediction for the human proteome. Nature. https://doi.org/10.1038/s41586-021-03828-1

Waman, V. P., Blundell, T. L., Buchan, D. W., Gough, J., Jones, D., Kelley, L., Murzin, A., Pandurangan, A. P., Sillitoe, I., \& Sternberg, M. (2020). The Genome3D Consortium for Structural Annotations of Selected Model Organisms. In Protein Structure Prediction (pp. 27-67). Springer.

Whittaker, R. H. (1969). New concepts of kingdoms of organisms. Science, 163(3863), 150160.

Williams, T. A., Cox, C. J., Foster, P. G., Szöllősi, G. J., \& Embley, T. M. (2020). Phylogenomics provides robust support for a two-domains tree of life. Nature ecology \& evolution, 4(1), 138-147. https://doi.org/10.1038/s41559-019-1040-x

Woese, C. R. (1987). Bacterial evolution. Microbiological reviews, 51(2), 221.

Woese, C. R., Kandler, O., \& Wheelis, M. L. (1990). Towards a natural system of organisms: Proposal for the domains Archaea, Bacteria, and Eucarya. Proceedings of the National Academy of Sciences of the United States of America, 87(12), 4576-4579.

Zardecki, C., Shao, C., Voigt, M., \& Burley, S. (2021). Protein Data Bank: 50 Years of Macromolecular Structures Enabling Research and Education. The FASEB Journal, 35.

Zaremba-Niedzwiedzka, K., Caceres, E. F., Saw, J. H., Bäckström, D., Juzokaite, L., Vancaester, E., Seitz, K. W., Anantharaman, K., Starnawski, P., Kjeldsen, K. U., Stott, M. B., Nunoura, T., Banfield, J. F., Schramm, A., Baker, B. J., Spang, A., \& Ettema, T. J. G. (2017). Asgard archaea illuminate the origin of eukaryotic cellular complexity [Article]. Nature, 541(7637), 353-358. https://doi.org/10.1038/nature21031 
Page 17 of 17 